\title{
Specific inhibition of NLRP3 comes closer
}

The past few years have seen an explosion of interest in the role of the NOD, LRR and pyrin

\section{CY-09}

prevented neonatal death

in a mouse

model of

Muckle-Wells

syndrome

(a cryopyrin-

associated

periodic

syndrome)

domain-containing 3 (NLRP3)

matory diseases and rheumatic diseases. Several inhibitors of the unwanted off-target effects. Now, the latest report in a long line of NLRP3. inflammasome in both autoinflamNLRP3 inflammasome are currently being developed as potential therapeutics, but some compounds have studies showcases a small-molecule inhibitor that is highly specific for

"Although both the components of the NLRP3 inflammasome and the related signalling events can be targeted to inhibit NLRP3 inflammasome activation, only directly targeting NLRP3 itself can specifically inhibit the NLRP3 inflammasome," states corresponding author Rongbin Zhou. "To look for a direct NLRP3 inhibitor, we first screened compound libraries, then excluded the compounds that can inhibit NF- $\kappa B$ or the activation of other inflammasomes, or that have effects on the upstream signalling events of NLRP3, including mitochondrial damage, potassium efflux and chloride efflux."

Zhou and colleagues found that the resulting molecule, CY-09, specifically bound to the ATP-binding motif of NLRP3 and inhibited its ATPase activity, blocking assembly of the NLRP3 inflammasome. In vivo, CY-09 prevented neonatal death in a mouse model of Muckle-Wells syndrome (a cryopyrin-associated periodic syndrome) and reduced insulin insensitivity in mice fed a high-fat diet (a model of type 2 diabetes mellitus). CY-09 also inhibited caspase- 1 activation and IL- $1 \beta$ production ex vivo in cells isolated from the synovial fluid of patients with gout.

"At this point in the field of NLRP3 inhibitors, the next question is whether the concentrations, which are effective in vitro and are used in mice, are safe and effective in humans," comments Charles Dinarello, who was not involved in the study. "Specific NLRP3 inhibitors have the potential to treat acute diseases such as gout flares, but also,

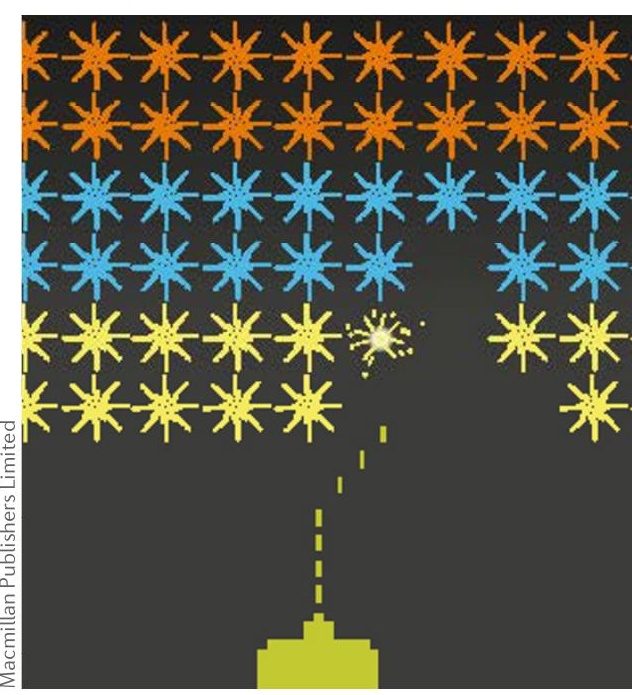

more importantly, chronic diseases such as heart failure, atherosclerosis and Alzheimer disease; however, CY-09 is a long way off from clinical reality," he concludes.

Joanna Collison

ORIGINAL ARTICLE Jiang, H. et al. Identification of a selective and direct NLRP3 inhibitor to treat inflammatory disorders. J. Exp. Med. http://dx.doi. org/10.1084/jem.20171419 (2017) 